\title{
Selective Excitation of Two-pulse Femtosecond Coherent Anti-Stokes Raman Scattering in a Mixture
}

\author{
Hui Zhang ${ }^{1}$, Xianghua Feng ${ }^{1}$, Zhongqiu $\mathrm{Yu}^{1}, \mathrm{Xia} \mathrm{Li}^{1}$, Shian Zhang ${ }^{2}$, Zhenrong Sun ${ }^{2}$ \\ ${ }^{1}$ Institute of Science, PLA Information Engineering University, Zhengzhou, China \\ ${ }^{2}$ State Key Laboratory of Precision Spectroscopy, East China Normal University, Shanghai, China
}

Received 2013

\begin{abstract}
In this paper, we experimentally study the selective excitation of two-pulse femtosecond coherent anti-Stokes Raman scattering (CARS) in a mixture of dibromomethane $\left(\mathrm{CH}_{2} \mathrm{Br}_{2}\right)$ and chloroform $\left(\mathrm{CHCl}_{3}\right)$ by adaptive pulse shaping based on genetic algorithm. Second harmonic generation frequency-resolved optical gating (SHG-FROG) traces indicate that the spectral amplitude and phase of the optimal pulse are both modulated. Finally, we discuss the physical mechanism for the selective excitation of femtosecond CARS based on the retrieved information from SHG-FROG traces.
\end{abstract}

Keywords: Coherent Anti-Stokes Raman Scattering; Selective Excitation; Pulse Shaping

\section{Introduction}

Coherent anti-Stokes Raman scattering (CARS), a nonlinear four-wave-mixing (FWM) process, is one of the most important nonlinear spectroscopic techniques, [1-5] and has been widely employed to investigate the molecular dynamical process. The selective excitation of special Raman mode can enhance the CARS spectroscopic contrast, and therefore it can be applied on microscopic investigation of complex molecular structure. However, for femtosecond CARS process, several Raman modes falling within the broad spectrum of the femtosecond pulses can be simultaneously excited, and it results in the poor selectivity between the different Raman modes. A promising method for the mode-selective excitation is coherent control by the femtosecond pulse shaping technique. Here, the femtosecond pulse is shaped by modulating the spectral amplitude and/or phase to control the light-matter interaction and achieve the desired outcomes. So far, coherent control by shaping the femtosecond pulse has been widely utilized to realize the selective excitation of femtosecond CARS. [6-19] Such as, the shaped femtosecond pulse with the simple spectral phase pattern of $\pi$-step, sinusoidal, chirped or binary function has been employed to realize the selective excitation of the femtosecond CARS. [8-14] Moreover, the use of adaptive feed- back control based on genetic algorithm or evolution strategy to selectively excite the femtosecond CARS has also been reported.[15-19]

In our previous study on the selective excitation of one or more Raman modes in the benzene $\left(\mathrm{C}_{6} \mathrm{H}_{6}\right)$ solution by the optimal control of two-pulse femtosecond CARS,[19] it was demonstrated that both the spectral amplitude and phase of the optimal pump and probe pulses are strongly modulated. In this paper, we further explore the physical control processes. We experimentally study the selective excitation of two-pulse femtosecond coherent anti-Stokes Raman scattering (CARS) in a mixture of dibromomethane $\left(\mathrm{CH}_{2} \mathrm{Br}_{2}\right)$ and chloroform $\left(\mathrm{CHCl}_{3}\right)$ by shaping femtosecond laser pulse based on genetic algorithm, the CARS signal from chloroform can be enhanced and simultaneously the CARS signal from dibromomethane is effectively suppressed. The original and optimal laser pulses are characterized by second harmonic generation frequency-resolved optical gating (SHG-FROG) technique, and finally the physical control mechanisms are explicitly discussed and analyzed based on the retrieved information from SHG-FROG traces.

\section{Experiment Setup}

The layout of the experimental arrangement is shown in Figure 1. A Ti:sapphire mode-locked laser (Spectra-Physics Spitfire amplifier) is used as the excitation source with the pulse duration of about $50 \mathrm{fs}$ and the center wavelength of $800 \mathrm{~nm}$. The output laser pulse is split into two components. One is used to pump an optical parametric amplifier (OPA) to generate the Stokes pulse. The other, as the pump and probe pulses, is sent into a programmable $4 \mathrm{f}$ pulse shaper. The pulse shaper is composed of a pair of diffraction gratings with 1200 lines/mm and a pair of concave mirrors with $200 \mathrm{~mm}$ focal length. A onedimensional programmable liquid-crystal spatial light modulator array (SLM-256, CRI) is placed at the Fourier 
plane of the shaper and used as updatable filter for the spectral amplitude and phase modulation. The frequency difference of the pump and Stokes pulses is set between the Raman shifts of dibromomethane and chloroform, and the dibromomethane and chloroform are mixed by the volume ratio $1: 1$. Their CARS signal is detected by the spectrometer equipped with a charge coupled device (CCD). A computer is served for reading the CARS signal, evaluating the cost function and updating the SLM. The original and optimal pump and probe pulses are characterized by second harmonic generation frequencyresolved optical gating (SHG-FROG) technique.[20]

\section{Results and Discussion}

Figure 2(a) shows CARS spectrum for the mixture of dibromomethane and chloroform excited by the original pulse. Two Raman signals are observed and labeled peak 1 (intensive) and peak 2 (weak). The peak 1 can be contributed to the Raman mode of dibromomethane at 1388 $\mathrm{cm}^{-1}$ and the peak 2 can be attributed to the Raman mode of chloroform at $1889 \mathrm{~cm}^{-1} \cdot[21,22]$

Our experimental goal is to enhance the weak CARS signal 2 from chloroform and maximally suppress the intense CARS signal 1 from dibromomethane. In our experiment, an optimal feedback control method based on genetic algorithms is used to selectively excite special Raman mode from the mixture of dibromomethane and chloroform. Genetic algorithms allow a change in all of the pixels of the spectral phase mask and a decision whether the change is either accepted or rejected according to the calculated fitness. Firstly, the initial voltage values are generated randomly and loaded on the pixels of SLM as the first generation. Secondly, the fitness for the contrast ratio of the CARS signal intensity from chloroform to that from dibromomethane is calculated, and then the voltage values of the new spectral mask for the second generation are generated by genetic algorithm operation (select, crossover and mutate). Finally, the abovementioned optimization procedure is repetitively performed till the

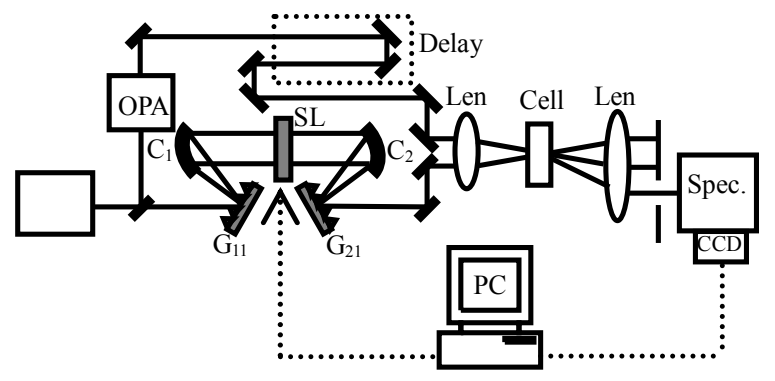

Figure 1. The experimental arrangement for selective excitation in stimulated Raman scattering. The programmable liquid-crystal spatial light modulator (SLM-256) is used as an updatable filter for spectral manipulation of the incident pulses. The computer is used for calculating the cost funtion and updating the spectral filter of the SLM. fitness approaches convergence and the contrast ratio approaches to the optimal value. Figure 2(b) shows CARS spectrum for the mixture of dibromomethane and chloroform excited by the optimal pulse. It is found that, Peak 1 from dibromomethane can be suppressed by one order of magnitude, but the Peak 2 from chloroform can be effectively enhanced by a factor of $\sim 8$.

To experimentally indicate the physical control process, second harmonic generation frequency resolved optical gating (SHG-FROG) technique is employed to characterize the original and optimal pump and probe pulses, and the experimental results are shown in Figure 3. The SHG-FROG traces show that the optimal pulse is strongly modulated. To further achieve the spectral information, the amplitude and phase of the original and optimal pulses in frequency and time domain are calculated based on the SHG-FROG traces and presented in Figure 4. As can be seen, the low wavelength components in optimal pulse are attenuated and simultaneously their phases are modulated (see Figure 4(c)). Moreover, the optimal pulse becomes pulse train (see Figure 4(d)). So we reasonably believe that the shaped pump and probe pulses with the combined amplitude and phase modulation should be the optimal field for the selective excitation of the two-pulse femtosecond CARS.
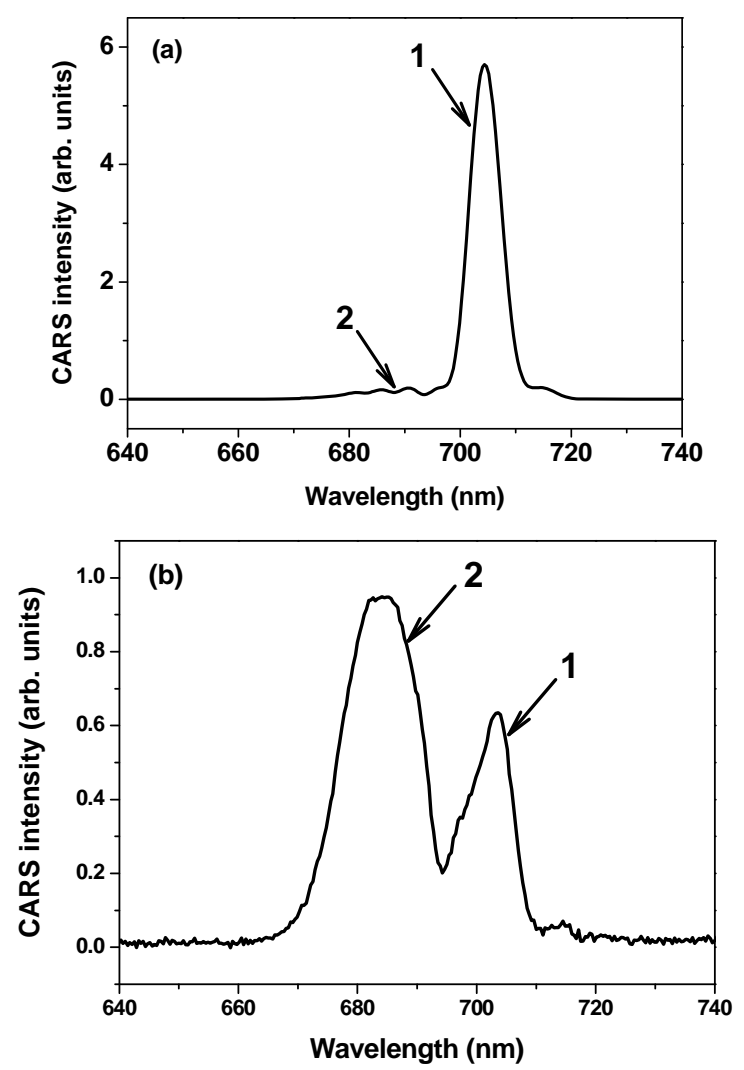

Figure 2. Stimulated Raman spectrum for the mixture of dibromomethane and chloroform is excited by the original pulse (a) and the optimal pulse (b). 

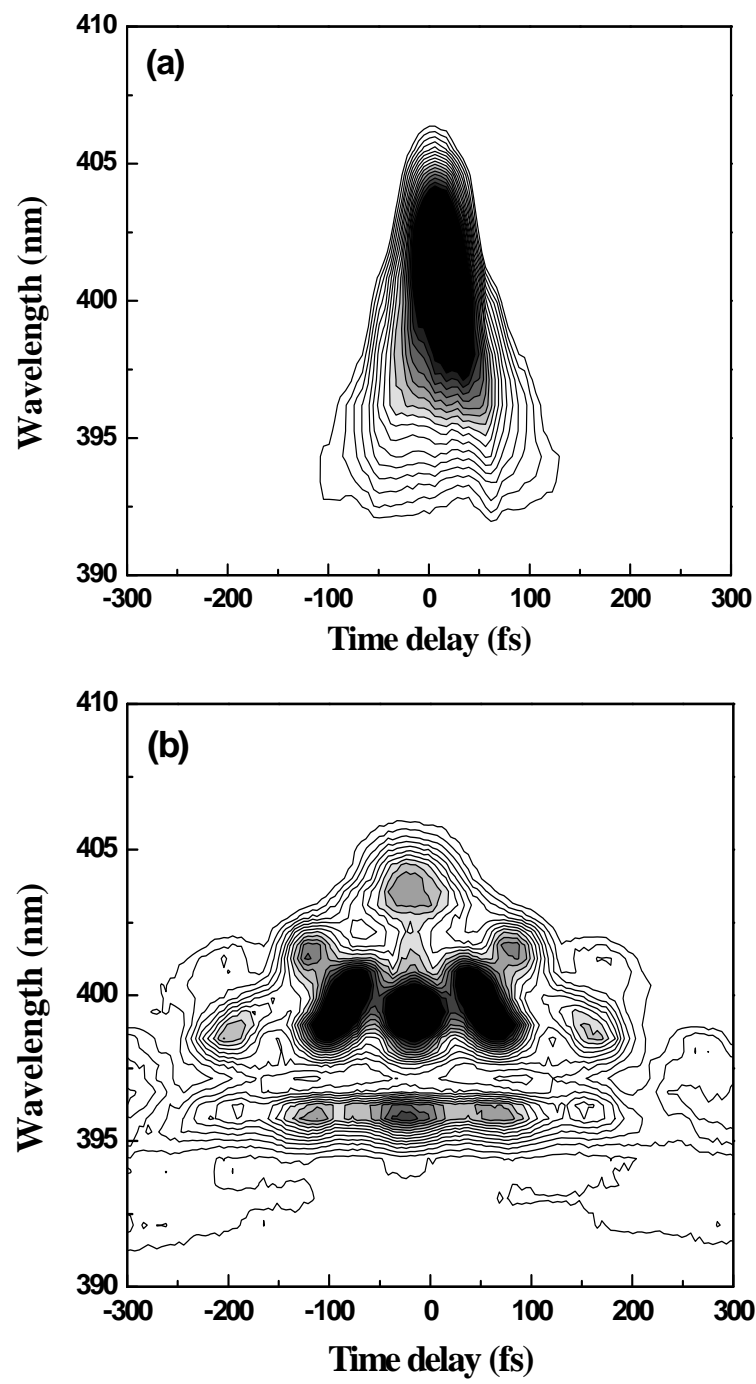

Figure 3. Second harmonic generation frequency resolved gating (SHG-FROG) traces for the original (a) and optimal pump pulse (b).

Next we discuss the physical mechanism for the selective excitation of femtosecond CARS based on the optimal pulse characteristic. According to the theory of Malinovskaya and Bucksbaum,[23] the stimulated Rman transition can be suppressed by tailoring its corresponding excitation frequency components. In our experiment, it is obvious that the peak 1 is induced by the blue frequency components of the pulse and the peak 2 is induced by the red frequency components. The blue frequency components of the optimal pulse are tailored (see Figure 4(c)), which results in the peak 1 being suppressed. Furthermore, the optimal pulse is modulated into pulse train (see Figure 4(d)), so the Stokes pulse will be prior to some sub-pulses in the pulse train, thus a stimulated Raman adiabatic passage (STIRAP) process can generate.[24,25] In this case, more population can transfer, which results in the peak 2 being enhanced.
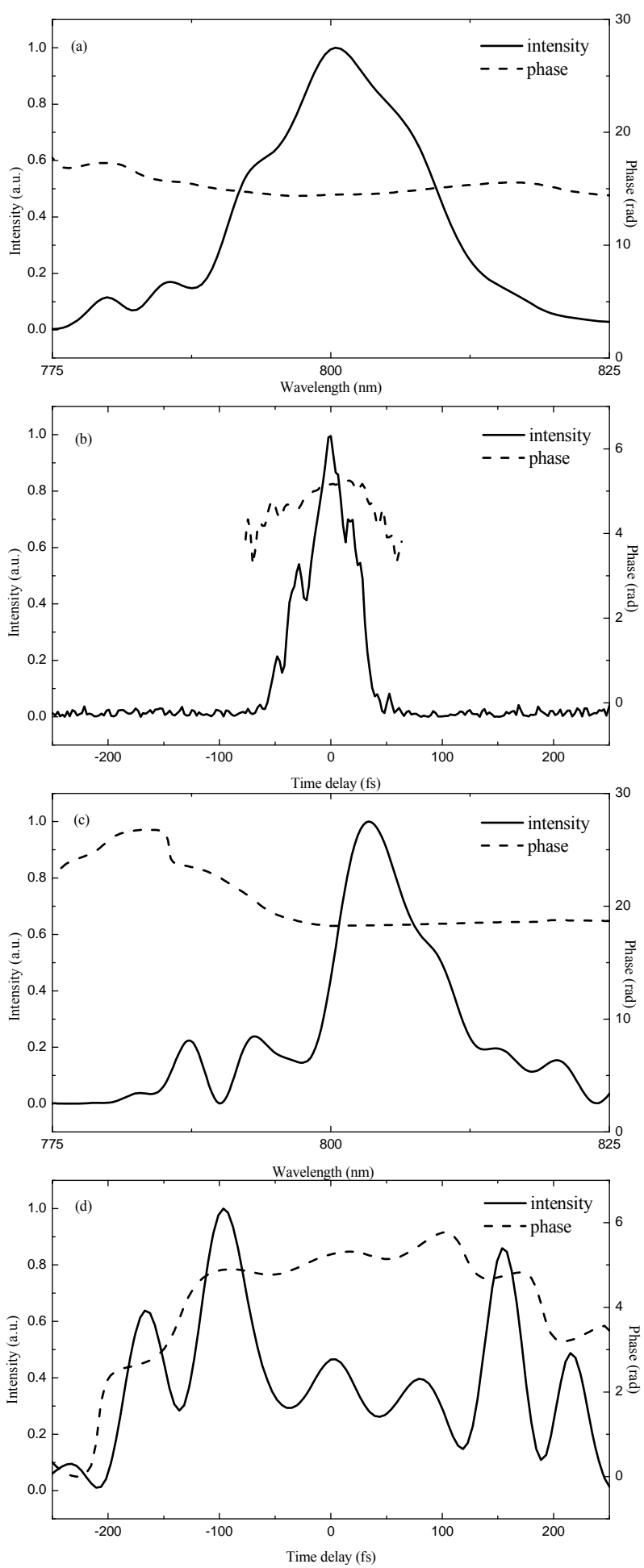

Figure 4. The amplitude and phase for the original (a,b) and optimal pulse (c,d) in frequency and time domains.

\section{Conclusions}

In summary, we have experimentally demonstrated the selective excitation of CARS in the mixture of dibromomethane $\left(\mathrm{CH}_{2} \mathrm{Br}_{2}\right)$ and chloroform $\left(\mathrm{CHCl}_{3}\right)$ by 
adaptive pulse shaping based on genetic algorithm. Second harmonic generation frequency-resolved optical gating (SHG-FROG) traces indicate that the spectral amplitude and phase of the optimal pulse are strongly modulated. Based on the retrieved information from SHG-FROG traces, it was proposed that the selective excitation of femtosecond CARS was due to laser spectrum tailored and pulse-train forming. We believe that these experimenttal results should have potential applications on stimulated Raman spectrum.

\section{REFERENCES}

[1] T. Chen, A. Vierheilig, P. Waltner, M. Heid, W. Kiefer and A. Materny, "Femtosecond Laser-Controlled Selective Excitation of Vibrational Modes on A Multidimensional Ground State Potential Energy Surface," Chemical Physics Letters, Vol. 326, 2000, pp. 375-382. doi:10.1016/S0009-2614(00) 00812-5

[2] M. Hashimoto, T. Araki and S. Kawata, "Molecular Vibration Imaging in the Fingerprint Region by Use of Coherent Anti-Stokes Raman Scattering Microscopy with A Collinear Configuration," Optics Letters, Vol. 25, No. 24, 2000, pp. 1768-1770. doi:10.1364/OL.25.001768

[3] F. El-Diasty, "Coherent Anti-Stokes Raman Scattering: Spectroscopy and Microscopy," Vibrational Spectroscopy, Vol. 55, 2011, pp. 1-37. doi:10.1016/j.vibspec.2010.09.008

[4] M. Lütgens, S. Chatzipapadopoulos and S. Lochbrunner, "Coherent Anti-Stokes Raman Scattering with Broadband Excitation and Narrowband Probe," Optics Express, Vol. 20, 2012, pp. 6478-6487. doi:10.1364/OE.20.006478

[5] D. D. Yavuz and Z. J. Simmons, "Optical Imaging with Nanoscale Resolution Using Optical Nonlinearities and Spatiotemporal Modulation," Physical Review A, Vol. 86, 2012, pp. 013817-013823. doi:10.1103/PhysRevA.86.013817

[6] Y. Silberberg, "Quantum Coherent Control for Nonlinear Spectroscopy and Microscopy," Annual Review of Physical Chemistry, Vol. 60, 2009, pp. 277-292. doi:10.1146/annurev.physche m.040808.090427

[7] C. Brif, R. Chakrabarti and H. Rabitz, "Control of Quantum Phenomena: Past, Present and Future,” New Journal for Physics, Vol. 12, 2010, pp.1-68. doi:10.1088/1367-2630/12/7/075008

[8] D. Oron, N. Dudovich, D. Yelin and Y. Silberberg, "Quantum Control of Coherent Anti-Stokes Raman Processes," Physical Review A, Vol. 65, 2002, pp. 043408-043411. doi:10.1103/PhysRevA.65.043408

[9] D. Oron, N. Dudovich, D. Yelin and Y. Silberberg, "Narrow-Band Coherent Anti-Stokes Raman Signals from Broad-Band Pulses," Physical Review Letters, Vol. 88, 2002, pp. 063004-063007. doi:10.1103/PhysRevLett.88.06300 4

[10] V. V. Lozovoy, B. Xu, J. C. Shane and M. Dantus, "Selective Nonlinear Optical Excitation with Pulses Shaped by Pseudorandom Galois Fields," Physical Review A, vol. 74,2006,pp. 041805-041808. doi:10.1103/PhysRevA.74.041805

[11] S. Malinovskaya, "Chirped Pulse Control Methods for Imaging of Biological Structure and Dynamics," International Journal of Quantum Chemistry, Vol. 107, 2007, pp. 3151-3158. doi:10.1002/qua.21513

[12] H. Li, D. A. Harris, B. Xu, P. J. Wrzesinski, V. V. Lozovoy and M. Dantus, "Coherent Mode-Selective Raman Excitation Towards Standoff Detection," Optics Express, Vol. 16, 2008, pp. 5499-5504. doi:10.1364/OE. 16.005499

[13] S. Pezeshki, M. Schreiber and U. Kleinekathöfer, "Shaping Femtosecond Coherent Anti-Stokes Raman Spectra Using Optimal Control Theory," Physical Chemistry Chemical Physics, Vol. 10, No. 15, 2008, pp. 2058-2066. doi:10.1039/B 714268D

[14] K. Isobe, A. Suda, M. Tanaka, H. Hashimoto, F. Kannari, H. Kawano, H. Mizuno, A. Miyawaki and K. Midorikawa, "Single-Pulse Coherent Anti-Stokes Raman Scattering Microscopy Employing An Octave Spanning Pulse," Optics Express, Vol. 17, 2009, pp. 11259-11266. doi:10.1364/ OE.17.011259

[15] J. Konradi, A. Scaria, V. Namboodiri and A. Materny, "Application of Feedback-Controlled Pulse Shaping for Control of CARS Spectra:The Role of Phase and AmpliTude Modulation," Journal of Raman Spectroscopy, Vol. 38, 2007, pp. 1006-1021. doi:10.1002/jrs.1701

[16] S. Zhang, L. Zhang, X. Zhang, L. Ding, G. Chen, Z. Sun and Z. Wang, "Selective Excitation of CARS by Adaptive Pulse Shaping Based on Genetic Algorithm," Chemical Physics Letters, Vol. 433, 2007, pp. 416-421. doi:10.1016/j.cplett. 2006.11.060

[17] J. Konradi, A. Gaál, A. Scaria, V. Namboodiri and A. Materny, "Influence of Electronic Resonances on Mode Selective Excitation with Tailored Laser Pulses," Journal of Physics Chemisty A, Vol. 112, 2008, pp. 1380-1391. doi:10.1021/jp 0745079

[18] A. Scaria, J. Konradi, V. Namboodiri and A. Materny, “A Comparison of the Selective Excitation of Molecular Modes in Gas and Liquid Phase Using Femtosecond Pulse Shaping," Journal of Raman Spectroscopy, Vol. 39, 2008, pp. 739-749. doi:10.1002/jrs. 1967

[19] P. J. Wrzesinski, D. Pestov, V. V. Lozovoy, B. Xu, S. Roy, J. R. Gord and M. Dantus, "Binary Phase Shaping for Selective Single-Beam CARS Spectroscopy and Imaging of Gas-Phase Molecules," Journal of Raman Spectroscopy, Vol. 42, 2011, pp. 393-398. doi:10.1002/jrs.2709

[20] K. W. DeLong, R. Trebino, J. Hunter and W. E. White, "Frequency-Resolved Optical Gating with the Use of Second-Harmonic Generation," Journal of the Optical Society of America (B), Vol. 11, 1994, pp. 2206-2215. doi:10.1364/JOSAB.11.002206

[21] R. S. Dennen, E. A. Piotrowski and F. F. Cleveland, "Raman and Infrared Spectral Data for $\mathrm{CH}_{2} \mathrm{Br}_{2}, \mathrm{CHDBr}_{2}$, and $\mathrm{CD}_{2} \mathrm{Br}_{2}$," Journal of Chemical Physics, Vol. 49, 1968, pp. 4385-4390. doi:10.1063/1.1669886 
[22] J. P. Zietlow, F. F. Cleveland and A. G. Meister, "Substituted Methanes. III. Raman Spectra, Assignments, and Force Constants for Some Trichloromethanes," Journal of Chemical Physics, Vol. 18, 1950, pp. 1076-1080. doi:10.1063/1.1747862

[23] S. A. Malinovskava, P. H. Bucksbaum and P. R. Berman, "Theory of Selective Excitation in Stimulated Raman Scattering," Physical Review A, Vol. 69, 2004, pp. 013801-013805. doi:10.1103/PhysRevA.69.013801
[24] K. Bergmann, H. Theuer and B. W. Shore, "Coherent Population Transfer Among Quantum States of Atoms and Molecules," Reviews of Modern Physics, Vol. 70, 1998, pp. 1003-1025. doi:10.1103/RevModPhys.70.1003

[25] J. Cheng, and J. Zhou, "Ultrafast Population Transfer in Three-Level $\Lambda$ Systems Driven by Few-Cycle Laser Pulses," Physical Review A, Vol. 64, 2001, pp. 065402-065405. doi:10.1103/ PhysRevA.64.065402 